\title{
LAS FAUNAS CUATERNARIAS DE MAMÍFEROS DE LA CUENCA DE BANYOLES-BESALÚ (GIRONA)
}

\author{
Ángel GALOBART ${ }^{1}$, Julià MAROTO² y Xavier ROS ${ }^{1}$ \\ 'Institut de Paleontología “M. Crusafont”, c/ Escola Industrial, 23. 08201 Sabadell. \\ 2 Aula de Prehistòria i Món Antic, Universitat de Girona, pl. Ferrater i Mora, 1. 17071 Girona / Centre d"Investigacions Arque- \\ ològiques, c/ Pedret, 95. 17071 Girona.
}

\begin{abstract}
Galobart, A., Maroto, J. y Ros, X. 1996. Las faunas cuaternarias de mamíferos de la cuenca de Banyoles-Besalú (Girona). [Quaternary Mammalia faunas from the Banyoles-Besalú basin (Girona)]. Revista Española de Paleontología, $\mathbf{N}^{\circ}$ Extraordinario, 248-255. ISSN 0213-6937.
\end{abstract}

\section{ABSTRACT}

\begin{abstract}
The Banyoles-Besalú Basin lacustrine deposits has yielded a great number of paleontological and archeological sites, with a well represented rich mammalian faunas of a perio between late Pliocene to recent Quaternary. In a important part of cases, this sites have been studied and described, specially the archeological ones, who are known until last years of the past century. In this paper are included all dates published and the new ones as a results of the revisions made for the doctoral thesis of two of the authors (A. G y J. M).
\end{abstract}

Keywords: Quaternary mammals, Banyoles-Besalú lacustrine basin.

\section{RESUMEN}

La cuenca lacustre de Banyoles-Besalú ha proporcionado un gran número de yacimientos paleontológicos y arqueológicos en los que se encuentra bien representada una rica fauna de mamíferos que abarca un período que se extiende desde finales del Plioceno hasta el Cuaternario reciente. En la mayoría de los casos estos yacimientos han sido ya estudiados y descritos, en especial los arqueológicos, conocidos desde finales del siglo XIX. En este trabajo se recogen las citas publicadas y se aportan nuevos datos como resultado de las revisiones que se están efectuado en las tesis doctorales de dos de los autores (A. G. y J. M).

Palabras clave: Mamíferos cuaternarios, cuenca lacustre Banyoles-Besalú.

\section{INTRODUCCIÓN}

La cuenca lacustre de Banyoles-Besalú ha sido profusamente estudiada. El interés estratigráfico de esta cuenca lacustre cuaternaria llevó a Ramon Julià (1980) a realizar su tesis doctoral, donde ya apuntaba la riqueza y variedad de la fauna fósil de mamíferos. Por otro lado los yacimientos arqueológicos de esta zona, conocidos desde el siglo pasado, han resultado ser de gran importancia por la continuidad y calidad de su registro. Las excavaciones realizadas en estos yacimientos tuvieron en cuenta la importancia de la fauna de mamíferos, así como otros vertebrados y restos antracológicos y palinológicos, permitiendo un ensayo de reconstrucción paleoambiental del entorno en el Paleolítico medio y superior.

Recientemente dos de los autores de este trabajo (A. G. y J. M.) han desarrollando sus tesis doctorales en dos de los más ricos conjuntos de esta cuenca: Incarcal y Bòbila Ordis -Pleistoceno inferior-, y parte de las cuevas paleolíticas del Reclau -Pleistoceno superior-. En el transcurso de estos trabajos se han contrastado las anteriores determinaciones faunísticas y aportado nuevas especies no contabilizadas hasta el momento.

\section{SITUACIÓN GEOGRÁFICA}

La cuenca lacustre de Banyoles-Besalú está situada a pocos kilómetros al norte de la ciudad de Girona, en la comarca del Pla de l'Estany (Fig. 1). Tiene una forma sub-rectangular de orientación aproximada N-S, y limita al N con los relieves de la Mare de Déu del Mont, al W y S con las estribaciones orientales de la Serralada Transversal y el límite $\mathrm{E}$, aunque es muy neto, no está marcado por ningún conjunto montañoso importante, sino por el cambio de paisaje de la depresión del Empordà.

\section{CONTEXTO GEOLÓGICO REGIONAL}

La cuenca lacustre de Banyoles-Besalú está delimitada por las unidades geomorfológicas de la Garrotxa, al oeste, y del Empordà, al este. Los materiales de la Garrotxa son de edad eocénica y constituyen un bloque levantado, mientras que los materiales detríticos del Empordà rellenan una fosa tectónica y son de edad neógena.

Dentro de esta cuenca se han desarrollado sucesivas etapas lacustres, con una sedimentación carbonatada y detrítica de materiales pliocuaternarios (Fig. 2). Se ha dividido en tres unidades geomorfológicas, que son de norte a sur: el glacis de Maià de Montcal, el llano de Usall y la cubeta lacustre de Banyoles (Julià, 1980).

En el glacis de Maià de Montcal afloran dos tipos de materiales; en la parte occidental dominan las facies detríticas groseras del glacis y en la parte oriental, las facies calcá- 


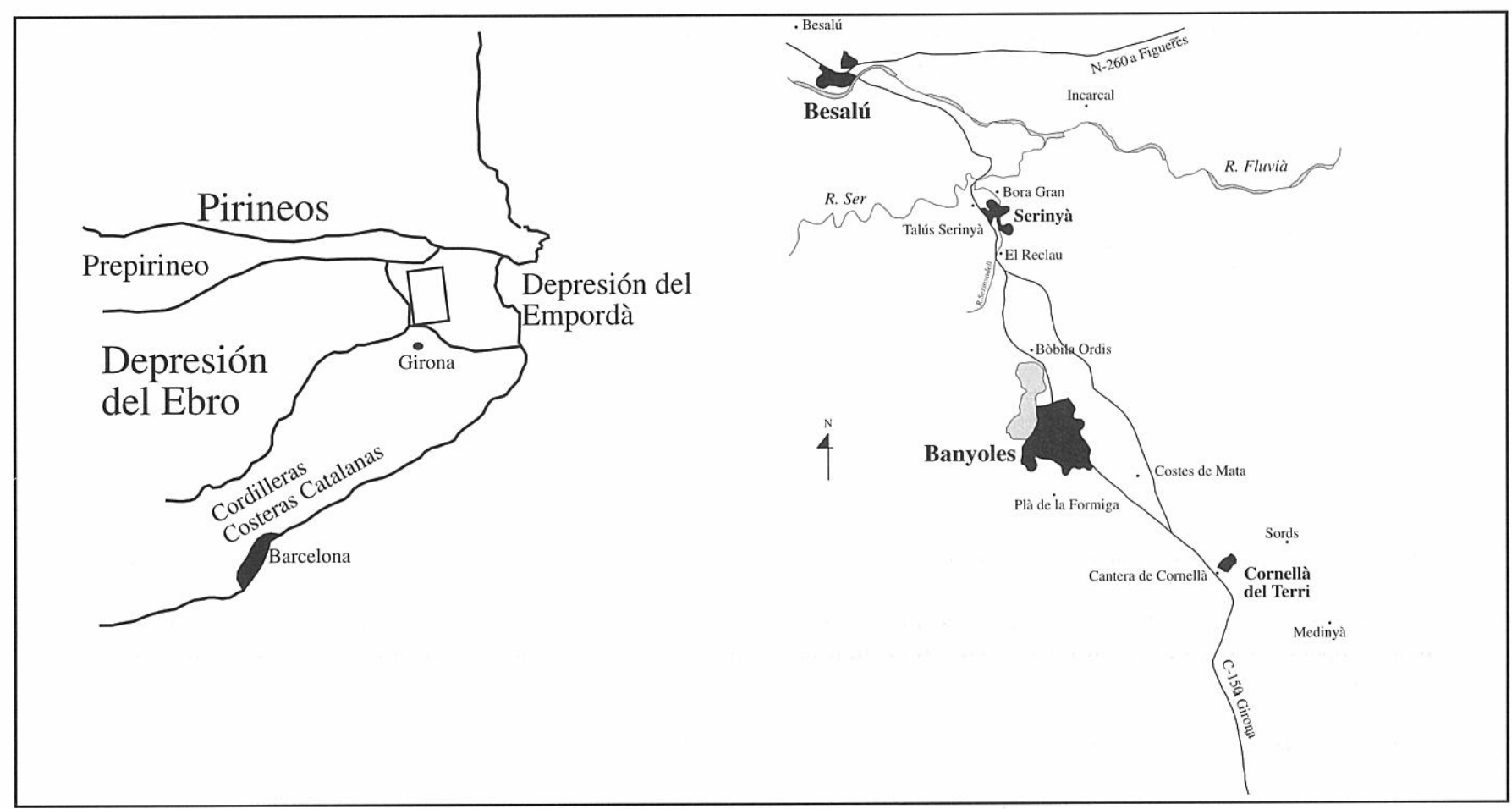

Figura 1. Contexto geológico regional y situación geográfica de la cuenca lacustre de Banyoles-Besalú. Situación de los yacimientos: 1. Besalú; 2. Incarcal; 3. Bora Gran; 4. Talús de Serinyà; 5. Cuevas del Reclau; 6. Bòbila Ordis; 7. Costes de Mata; 8. Pla de la Formiga; 9. Sords; 10. Cantera de Cornellà; 11. Medinyà.

reas (formación Incarcal). En estas calizas se encuentran embudos kársticos rellenados por arcillas en un ambiente lacustre, que contienen abundante fauna fósil.

El llano de Usall está formado por calizas lacustres, recubiertas por formaciones superficiales (suelos rojos, arcillas, limos y gravas) en su mitad meridional y en su mitad septentrional afloran sedimentos detríticos y localmente terrazas travertínicas (cuevas del Reclau).

La tercera unidad, la cubeta lacustre de Banyoles, está formada por diferentes tipos de travertinos, originados por el drenaje natural del lago de Banyoles, que constituyen el dique estromatolítico y la llanura de inundación.

\section{LA FAUNA}

\section{PLIOCENO}

Los fósiles procedentes de la cantera de Cornellà del Terri y de otras localidades situadas en los sedimentos detríticos neógenos de la depresión del Empordà (Medinyà, Costes de Mata y Sords) han proporcionado una escasa fauna de mamíferos de finales del terciario.

En el yacimiento de Cornellà del Terri, el más rico en cantidad y diversidad, se han recolectado fósiles desde 1950. Se trata de una explotación de arcillas donde esporádicamente se han hallado restos de mamíferos, fruto de hallazgos fortuitos de los trabajadores o de prospecciones de aficionados. La colección faunística todavía no se ha estudiado sistemáticamente.

La secuencia es totalmente terrígena, constituida por materiales detríticos -arcillas, arenas y microconglomera- dos- y depositada en un ambiente aluvial. Forma parte de los rellenos aluviales neógenos que colmatan la depresión del Empordà y corresponden a una etapa prelacustre (Fig. 2).

De un examen preliminar de estos restos presentamos la siguiente lista faunística, que ya habíamos avanzado en Sanz et al. (1987), compuesta actualmente por cinco taxones:

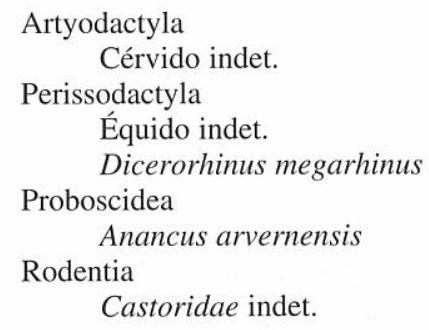

En este yacimiento la especie mejor representada corresponde al mastodonte Anancus arvernensis, con presencia de abundantes restos craneales: diversos fragmentos de defensa ( 3 enteras), algunos de mandíbula, tres molares superiores y dos inferiores. El resto de la fauna está escasamente representada, como es el caso del équido, por un molar inferior muy gastado que no permite asignarlo a Hipparion o a Equus; el de Dicerorhinus megarhinus, por un molar superior y un cérvido no determinado del que se conserva un fragmento de asta. Estos restos se encuentran depositados en el Museu Arqueològic Comarcal de Banyoles (M.A.C.B.).

En dos localidades se ha citado la presencia de Zygolophodon borsoni. En la localidad de Can Magrana, término municipal de Medinyà (Villalta y Llompart, 1981) y en las Costes de Mata (Julià, 1980; Villalta y Llompart, 1981). De 
la primera procede un magnífico molar, de propiedad particular (hay una copia depositada en la Universitat de Girona), descrito por Villalta y Llompart (1981). De la segunda, un molar muy fragmentado depositado en el M.A.C.B., descrito en Sanz et al. (1987).

Por último, tenemos otro molar superior de rinoceronte, muy bien conservado pero hoy desgraciadamente desaparecido del M.A.C.B., procedente de Sords, que hemos clasificado como Dicerorhinus megarhinus en Sanz et al. (1987).

Estas tres localidades también forman parte de los materiales aluviales del Empordà.

El contexto regional prelacustre y los taxones identificados nos permiten asignar estos yacimientos como pliocenos, sin que de momento podamos precisar más su cronología.

\section{PLEISTOCENO INFERIOR}

Los yacimientos de mamíferos fósiles del Pleistoceno inferior se hallan situados en sendas explotaciones comerciales de calizas y arcillas. Los trabajos de extracción de los sedimentos han permitido en ambos casos la recuperación de los restos fósiles. En Incarcal mediante una serie de campa-

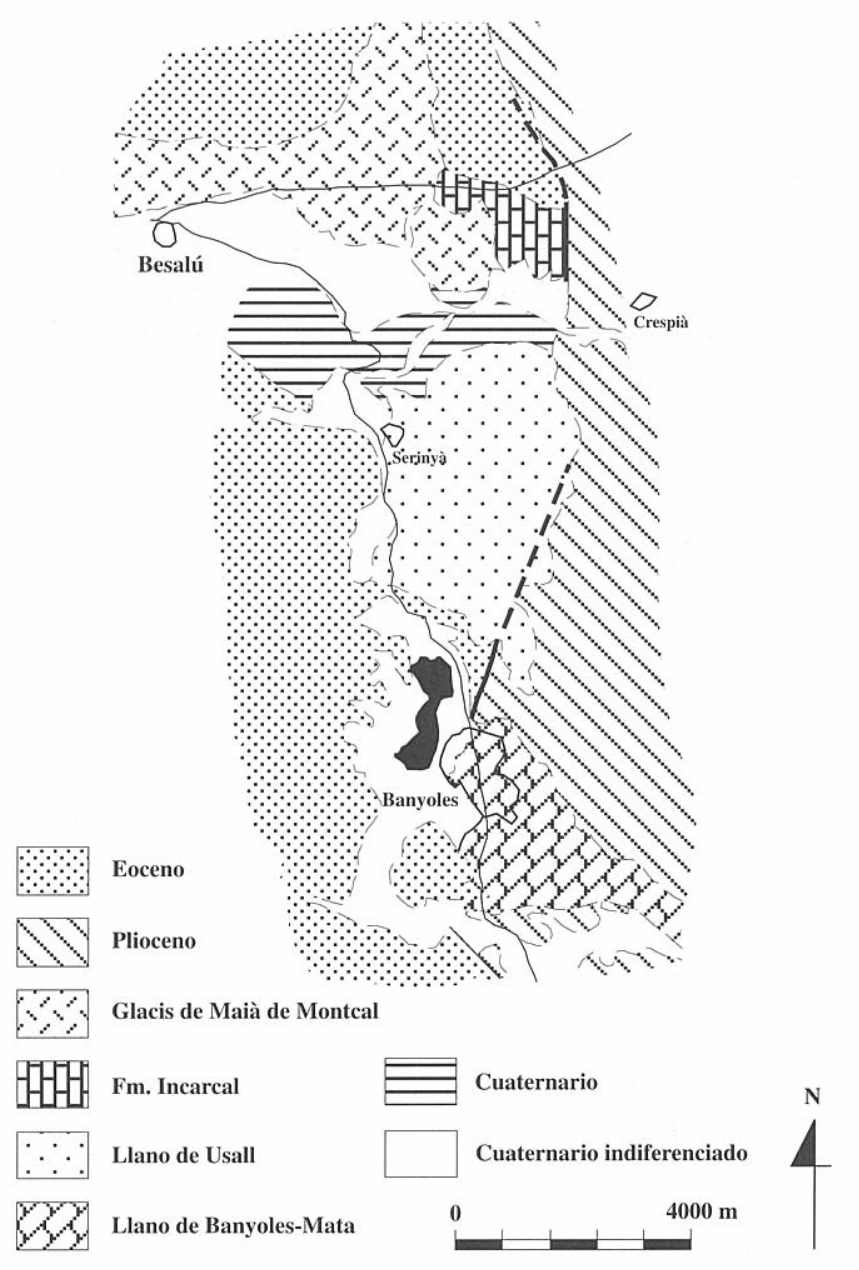

Figura 2. Mapa geológico de la cuenca de Banyoles-Besalú (modificado de Julià, 1980). ñas de excavaciones programadas, y en Bòbila Ordis por los hallazgos fortuitos y las donaciones al Museu Arqueològic Comarcal de Banyoles.

\section{Incarcal}

Incarcal es el yacimiento más importante de este periodo, tanto por el número de taxones que ha proporcionado como por su estado de conservación. Se encuentra al sur de la N-260, a pocos kilómetros de Besalú en dirección a Figueres.

La primera noticia de la presencia de fósiles en la cantera de Incarcal se remonta al año 1965, en que los encargados de la extracción de los carbonatos dan a conocer a E. Sanz, del Centre d'Estudis Comarcals de Banyoles, la presencia de restos en las bolsas de arcillas que afectan a las calizas lacustres. Los primeros estudios corresponden a J. F. Villalta y R. Julià que realizarían las primeras prospecciones de la zona (Julià y Villalta, 1974; Julià,1977, 1979, 1980; Deckker et al.,1979; Julià y Villalta, 1984).

Las calizas de Incarcal, con una potencia total de $70 \mathrm{~m}$, están constituidas por calcisiltitas muy blancas (Julià y Villalta, 1984). Se alternan las capas puras predominantes con otras de acumulaciones de fragmentos de caráceas. Localmente contienen abundantes hojas vegetales (Villalta y Vicente, 1972; Roiron, 1983) y muy esporádicamente restos óseos (Fig. 2).

El yacimiento está formado actualmente por una serie de nueve embudos, o dolinas, de formación kárstica, de desigual riqueza, que se encuentran incluidos en el substrato de calizas lacustres finipliocénico (Julià, 1980; Roiron, 1983). Las dolinas, formadas por disolución, están rellenadas básicamente de arcillas, con algunos niveles arenosos y microconglomeráticos, y abundantes restos de fauna.

La excavación sistemática de los yacimientos -especialmente de dos embudos- se inició en 1984 y finalizó en 1990, con un listado faunístico que ha ido aumentando a medida que avanzaban los trabajos de extracción y estudio de los materiales. Así, de las ocho especies de mamíferos citadas por Julià y Villalta (1984), se ha ido aumentando (Maroto y Soler, 1985; Galobart et al., 1990) hasta llegar a las dieciséis actuales (Colomer et al., 1992; Maroto y Galobart, 1992).

Insectivora
Sorex cf. minutus
Lagomorpha
Oryctolagus cf. lacosti
Prolagus cf. calpensis
Rodentia
Mimomys cf. blanci
Allophaiomys sp.
Apodemus aff. mystacinus
Carnivora
Pachycrocuta brevirrostris
Canis sp.
Homotherium crenatidens
Artiodactyla
Hippopotamus amphibius antiquus
Megaceros sp
Bos-Bison
cf. Capreolus
Perissodactyla




\author{
Stephanorhinus etruscus \\ Equus stenonis \\ Proboscidea \\ Archidiskodon meridionalis
}

De la lista aquí expuesta cabe reseñar la mayor abundancia relativa de restos de carnívoros sobre los de herbívoros, y el excelente grado de conservación de los fósiles.

El félido de dientes de sable, Homotherium crenatidens es, con mucho, la especie mejor representada cualitativamente. Sus cuatro cráneos y numerosos restos postcraneales están permitiendo una precisa descripción de esta especie común en el Pleistoceno inferior europeo. Asimismo, otras especies bien representadas son el hipopótamo (Hippopotamus amphibius antiquus), el elefante meridional (Archidiskodon meridionalis) y la hiena (Pachycrocuta brevirostris), con una gran variedad y cantidad de restos óseos craneales y postcraneales.

Por el momento no disponemos de ninguna datación absoluta que nos permita corroborar los resultados obtenidos de la propia fauna.

\section{Bòbila Ordis}

A la salida de Banyoles, en dirección a Olot por la carretera C-150, se encuentra la cantera de arcillas de Bòbila Ordis. Este yacimiento ha sido objeto de estudio por parte de diversos autores. Julià $(1977,1979,1980)$ y Julià y Suc (1980) describen el yacimiento y sus hallazgos faunísticos y palinológicos.

Las arcillas de Bòbila Ordis fueron depositadas en un medio lacustre y rellenan una pequeña y profunda cubeta. Presentan diferentes facies y el hecho que hayan sido objeto de explotación continuada dificulta mucho la localización estratigráfica de los hallazgos. Se interpretan como pertenecientes al pleistoceno inferior en base a su contenido faunístico. Los análisis palinológicos y paleomagnéticos efectuados no contradicen esta edad.

El material faunístico recuperado se encuentra muy fragmentado en la mayoría de los casos; nos ha permitido determinar hasta el momento un total de nueve especies:

$$
\begin{aligned}
& \text { Rodentia } \\
& \text { Mimomys savini } \\
& \text { Carnivora indet. } \\
& \text { Artiodactyla } \\
& \text { Hippopotamus amphibius } \\
& \text { Megaceros sp. } \\
& \text { Bos-Bison } \\
& \text { Cérvido de pequeña talla } \\
& \text { Perissodactyla } \\
& \text { Stephanorhinus etruscus } \\
& \text { Equus stenonis } \\
& \text { Proboscidea } \\
& \text { Archidiskodon meridionalis }
\end{aligned}
$$

J. Agustí (comunicación personal) determinó la única especie de micromamífero obtenido hasta el momento, el arvicólido Mimomys savini.

\section{PLEISTOCENO MEDIO}

Dos localidades han sido las que han aportado materiales del Pleistoceno medio en la cuenca. Por un lado, el hallaz- go de restos muy fragmentados de un proboscídeo en la localidad de Besalú, y por otro, la fauna de los niveles inferiores de la cueva de Mollet I en el paraje del Reclau.

\section{Besalú}

A la salida de la población de Besalú en dirección a Olot se recuperaron, en los materiales detríticos del glacis de Maià de Montcal (Fig. 2), unos restos muy fragmentados de un proboscídeo.

De todos ellos sólo hemos podido identificar una pequeña parte, entre los que destacan un fragmento de molar, varias epífisis de huesos largos y una diáfisis de húmero.

Este material no nos permite una determinación específica concreta y sólo podemos constatar un tamaño menor al Archidiskodon meridionalis de Incarcal, y un rasgo dentario que Aguirre (1969) califica de arcaico. Este rasgo consiste en la presencia de islas de esmalte en los valles transversales del molar, carácter observable con cierta frecuencia en A. meridionalis y, de forma más restringida, en formas arcaicas de Elephas antiquus.

El glacis de Maià de Montcal recubre discordantemente a los embudos de Incarcal. Este dato, más la presencia de los restos de elefante, nos induce a pensar en una edad mesopleistocénica sensu lato.

\section{Cueva de Mollet}

Situada en el conjunto de cuevas prehistóricas del Reclau (Serinyà), los trabajos de excavación de este yacimiento se llevaron a término por Josep M. Corominas en cuatro etapas entre 1947 y 1972.

Anna Mir estudió la fauna del yacimiento (Mir y Salas, 1976; Mir, 1979). En Maroto et al. (1987) se completa la lista faunística que corresponde a los estratos inferiores. Esta lista, revisada la nomenclatura, es la siguiente:

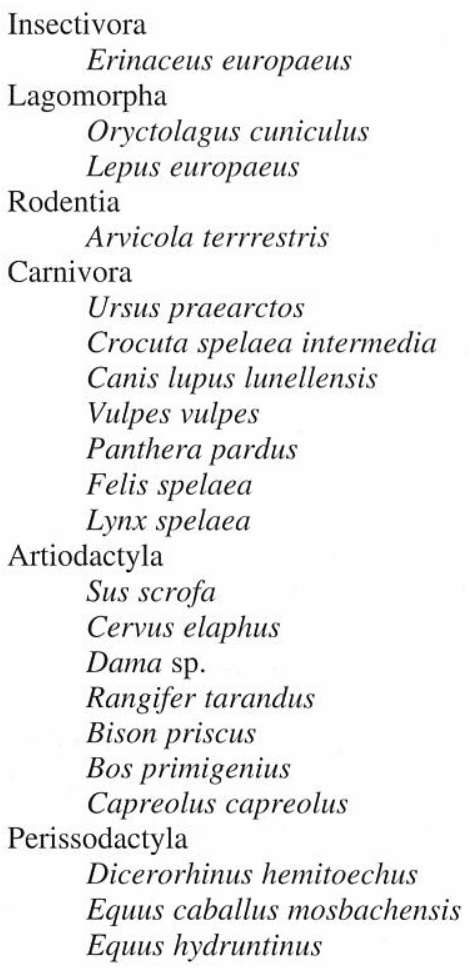




\author{
Proboscidea \\ Hominidae \\ Elephas antiquus
}

Homo sapiens ssp.

Se constata la presencia humana por el hallazgo de un primer molar superior, de un individuo muy joven, de una forma anteneandertal o neandertal (Cortada y Maroto, 1990).

Mir y Salas (1976) y Maroto et al. (1987) compararon esta fauna con la de los yacimientos de Lunel-Viel, L'Escale y Observatoire de Mónaco, y coincidieron en que se habría depositado en un momento prewürmiense.

Destaca entre los restos de Mollet la presencia de dos carnívoros (Canis lupus lunellensis y Crocuta spelaea intermedia) claramente mesopleistocénicos, y de un tercero (Ursus praearctos) que puede ser del pleistoceno inferior o del pleistoceno medio y que también ha sido estudiado por $\mathrm{T}$. de Torres (1988). Esta fauna está asociada a una industria musteriense de talla levallois, que nos permite situar al conjunto en una fase no antigua del Pleistoceno medio.

\section{PLEISTOCENO SUPERIOR}

Las faunas de mamíferos del Pleistoceno superior se encuentran localizadas en cuatro puntos de la cuenca: las cuevas del Reclau, la Bora Gran, el talud de Serinyà y los travertinos de Banyoles.

\section{Cuevas del Reclau}

El conjunto de las cavidades del Reclau (Arbreda, Arbreda II, Mollet -de la que acabamos de hablar-, Mollet III, Pau y Reclau Viver), descubiertas arqueológicamente y excavadas desde 1943 hasta 1973 por Josep M. Corominas, constituyen un excelente grupo de yacimientos del Paleolítico medio y superior que ha proporcionado una rica fauna. Los trabajos recientes de excavación han tenido lugar en la cueva de l'Arbreda entre 1975 y 1987, y han sido dirigidos por Narcís Soler y Julià Maroto.

\section{Cueva de la Arbreda}

La cueva de la Arbreda es la mayor y la más completa tanto en el registro arqueológico, que abarca del Musteriense hasta el Solutrense, como en el faunístico.

Los primeros trabajos sobre la fauna de la Arbreda corresponden a Estévez (1977-78, 1978, 1980,1985), en los cuales da un listado faunístico que corregirá y aumentará en un trabajo ulterior (Estévez, 1987). Posteriormente se ha ido completando este estudio (Casellas y Maroto, 1986; Soler y Maroto, 1987; Maroto, 1994).

En cuanto a los micromamíferos, G. Alcalde (Alcalde y Brunet-Lecomte, 1985; Alcalde, 1986; Alcalde, 1987) ha realizado el estudio de los roedores, el cual aun prosigue, y actualmente se está llevando a cabo por uno de nosotros (A. G.) el de los insectívoros y quirópteros, que nos ha permitido aumentar la lista de especies.

Esta lista de especies, repartida por niveles, queda en estos momentos de la siguiente manera.

\section{Nivel I (Musteriense reciente)}

Contiene una industria musteriense muy rica y típica, datada en $40.400 \pm 1.400 \mathrm{BP}$ (Bischoff et al., 1989).

Insectivora
Erinaceus europaeus
Chiroptera
Myotys myotis
Rodentia
Eliomys quercinus
Microtus arvalis-agrestis
Pitymys sp.
Arvicola sp.
Apodemus sylvaticus
Lagomorpha
Oryctolagus cuniculus
Lepus europaeus
Carnivora
Canis lupus
Vulpes vulpes
Ursus spelaeus
Crocuta spelaea
Lynx cf. spelaea
Felis sylvestris
Felis spelaea
Artyodactila
Bos-Bison
Cervus elaphus
Capreolus capreolus
Perissodactyla
Equus caballus
Equus hydruntinus

\section{Nivel H (Auriñaciense arcaico)}

Es un nivel correspondiente a un Auriñaciense inicial muy antiguo, pero a la vez muy característico. Está datado en $38.500 \pm 1.000$ BP (Bischoff et al., 1989).

Insectivora
Neomys fodiens-anomalus
Sorex minutus
Erinaceus europaeus
Rodentia
Eliomys quercinus
Microtus arvalis-agrestis
Pitymys sp.
Arvicola sp.
Apodemus sylvaticus
Chiroptera
Myotys myotis
Plecotus auritus-austriacus
Miniopterus schreibersi
Lagomorpha
Oryctolagus cuniculus
Lepus europaeus
Carnivora
Canis lupus
Vulpes vulpes
Ursus spelaeus
Crocuta spelaea
Lynx spelaea
Artyodactila
Bos-Bison
Rupicapra rupicapra




\author{
Cervus elaphus \\ Capreolus capreolus \\ Perissodactyla \\ Equus caballus \\ Proboscidea$$
\text { Equus hydruntinus }
$$ \\ Mammuthus-Elephas
}

Destaca la presencia de restos de elefante -que por la cronología parece que habría que atribuir con más probabilidad a mamut-, pero en realidad todos los restos pertenecen a unos pocos fragmentos de defensa trabajados antrópicamente para realizar útiles, con lo cual existe la posibilidad que fueran importados como materia prima.

\section{Nivel G (Auriñaciense evolucionado)}

Es un nivel muy rico arqueológicamente que contiene una industria correspondiente a un auriñaciense tardío. Está datado en $22.590 \pm 290$ BP (Delibrias et al., 1987).

Insectivora

Erinaceus europaeus

Sorex minutus

Sorex araneus

Crocidura rusula

Chiroptera

$$
\text { Myotis myotis }
$$

Lagomorpha

Oryctolagus cuniculus

Rodentia

Lepus europaeus

Glis glis

Eliomys quercinus

Microtus arvalis-agrestis

Pitymys sp.

Apodemus sylvaticus

Carnivora

$$
\text { Vulpes vulpes }
$$

Artyodactila

Cervus elaphus

Capreolus capreolus

Bos primigenius

Bison priscus

Capra pyrenaica

Perissodactyla

Equus caballus

Equus hydruntinus

\section{Nivel E y F (Gravetienses)}

Son niveles que contienen una industria gravetiense típica. El superior está datado en $20.130 \pm 220 \mathrm{BP}$ (Delibrias et al., 1987).

\section{Insectivora}

Erinaceus europaeus

Sorex minutus

Sorex araneus

Crocidura rusula

Chiroptera

Myotis myotis

Plecotus austriacus/auricus

Lagomorpha

Oryctolagus cuniculus

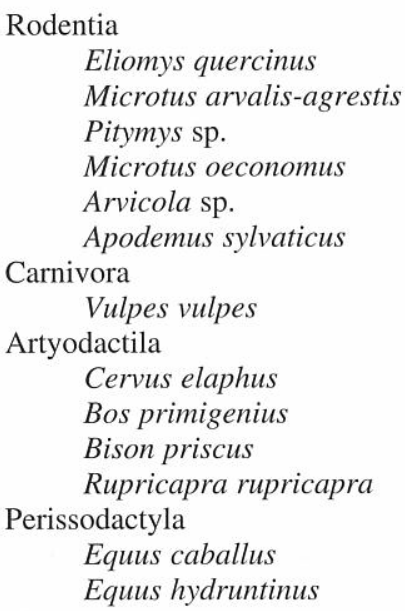

En esta lista, cabe subrayar la presencia de un micromamífero, Microtus oeconomus, cuya distribución actual es más septentrional.

\section{Niveles B, C y D (Solutrenses)}

Se trata de tres niveles que contienen industria solutrense. El C está datado en $17.320 \pm 290 \mathrm{BP}$ y el D en $17.720 \pm$ 290 BP (Delibrias et al., 1987).

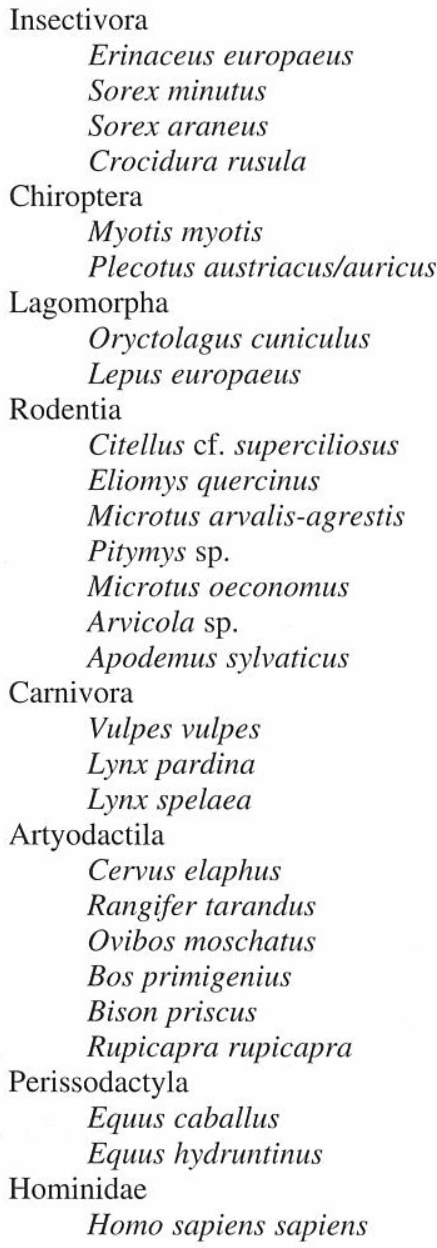

Citellus cf. superciliosus

Eliomys quercinus

Microtus arvalis-agrestis

Pitymys sp.

Microtus oeconomus

Arvicola sp.

Apodemus sylvaticus

Carnivora

$$
\begin{aligned}
& \text { Vulpes vulpes } \\
& \text { Lynx pardina }
\end{aligned}
$$

Lynx spelaea

Artyodactila

Cervus elaphus

Rangifer tarandus

Ovibos moschatus

Bos primigenius

Bison priscus

Rupicapra rupicapra

Perissodactyla

Equus caballus

Hominidae

Equus hydruntinus

Homo sapiens sapiens

Destacan las cuatro especies que son poco frecuentes en el Mediterráneo sudpirenaico, dos de macromamíferos y dos 
de micromamíferos. Las primeras son el Ovibos moschatus y el Rangifer tarandus, aunque esta última está representada solamente por una asta de muda utilizada por los solutrenses como percutor y por tanto cabe la probabilidad de que se trate de una pieza importada; el buey almizclado está presente por un tercer molar inferior (Estévez, 1977-78, 1978). Las segundas son el Citellus cf. superciliosus y el Microtus oeconomus (Alcalde, 1987).

\section{La Bora Gran d'en Carreras}

La Bora Gran d'en Carreras (Serinyà) es un magnífico abrigo abierto en conglomerados aluviales -presumiblemente cuaternarios (Fig. 2) - que ha proporcionado el que hasta el momento es el mejor yacimiento magdaleniense de Cataluña, y que por tanto nos muestra una interesante fauna antrópica finipleistocénica.

Los materiales recuperados proceden de excavaciones antiguas -Pere Alsius y Edouard Harlé a finales del siglo pasado, Isidre Bosoms a inicios del presente, Josep M. Corominas en los años 30 y Lluís Pericot y Joan Maluquer en los años 40 - y por tanto no presentan micromamíferos. Poseen una datación absoluta de $11.470 \pm 500$ años B.P. (Crane y Griffin, 1960; Corominas \& Marqués, 1967) que los sitúan en el magdaleniense superior y que no se contradice con la evidencia arqueológica. Los restos de mamíferos han sido estudiados de nuevo recientemente por Jordi Nadal, de cuyo estudio reproducimos la siguiente relación faunística (Nadal et al., en prensa).

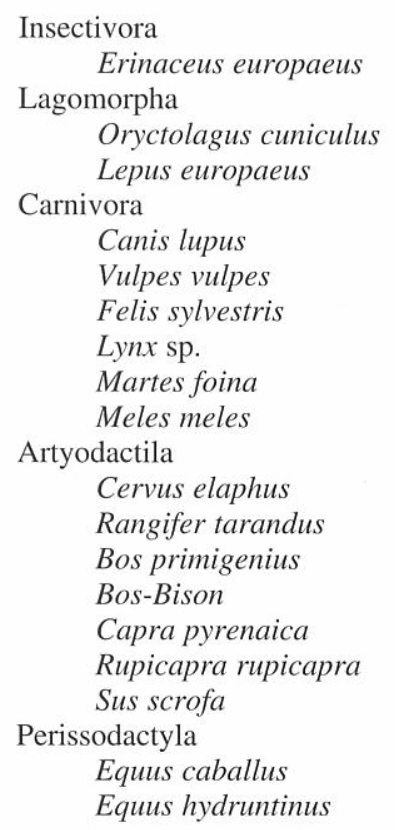

Destaca de nuevo la presencia del reno, aunque esta vez algún escaso resto postcraneal acompaña a los restos de asta.

\section{Talud de Serinyà}

Las obras de mejora de la carretera C-150 de Banyoles a Olot dejaron al descubierto un afloramiento que consistía en el relleno arcilloso de una grieta producida por la bascula- ción de los materiales detríticos encajantes - la misma unidad que forma la Bora Gran- (fig. 2).

En estas arcillas se han identificado cinco taxones de vertebrados.

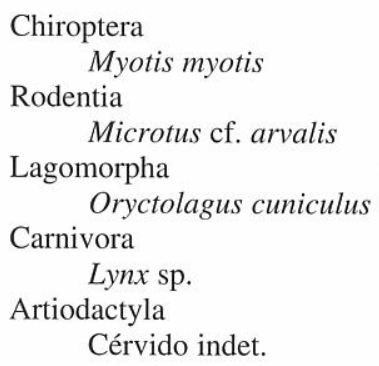

\section{Banyoles y Pla de Mata}

La fauna estudiada en los sedimentos travertínicos de Banyoles corresponde a un Pleistoceno superior. Las dataciones efectuadas por el momento para estos travertinos se sitúan entre 117.000 y 44.000 (Julià y Bischoff, 1991), pero con seguridad los hay más antiguos y más recientes.

Se han agrupado las diferentes localidades en un solo yacimiento que caracterizaría la fauna de la unidad geológica que es el llamado miembro Formiga (Julià, 1980, Julià et al., 1987).

Es en esta localidad donde se halló la mandíbula anteneandertal o neandertal de Banyoles (Maroto, 1993).

La fauna acompañante recogida desde finales del siglo pasado y citada por varios autores, hasta el momento corresponde, por lo que se refiere a los mamíferos, a un total de seis especies (Sanz et al., 1987).

$$
\begin{aligned}
& \text { Artiodactyla } \\
& \text { Cervus elaphus } \\
& \text { Bos primigenius } \\
& \text { Bison priscus } \\
& \text { Perissodactyla } \\
& \text { Equus caballus } \\
& \text { Equus hydruntinus } \\
& \text { Hominidae } \\
& \text { Homo sapiens ssp. }
\end{aligned}
$$

Asimismo, Sanz et al. (1987), citan diversas localidades que han proporcionado restos fósiles del Pleistoceno superior y del Holoceno en los travertinos de Banyoles.

\section{BIBLIOGRAFÍA}

Aguirre, E. 1969. Revisión sistemática de los Elephantidae por su morfología y morfometría dentaria (Primera parte). Estudios Geológicos, XXIV, 3-4, 109-167.

Alcalde, G. 1986. Les faunes de rongeurs du Pléistocène Supérieur et de l'Holocène de Catalogne (Espagne) et leurs significations paléoècologiques et paléoclimatiques, Diplome, École Practique des Hauts Études, Paris.

Alcalde, G. 1987. Els rosegadors del Paleolític superior de la cova de l'Arbreda (Serinyà, Catalunya). Significació paleoecològica i paleoclimàtica. Cypsela, VI, 89-96.

Alcalde, G. i Brunet-Lecomte, P. 1985. Contribució al coneixement del medi i el clima durant el Pleistocè superior i l'Holocè a 
Catalunya, amb l'aplicació de l'anàlisi factorial de correspondències a les associacions de rosegadors. Paleontologia $i$ Evolució, 19, 49-55.

Bischoff, J. L., Soler, N., Maroto, J. and Julià, R. 1989. Abrupt Mousterian/Aurignacian Boundary at c. $40 \mathrm{ka} \mathrm{bp}$ : Accelerator ${ }^{14} \mathrm{C}$ dates from L'Arbreda Cave (Catalunya, Spain). Journal of Archaeological Science, 16, 563-576.

Casellas, S. et Maroto, J. 1986. La faune de l'Aurignacien évolué de la grotte de l'Arbreda (Girona, Espagne), Résumés de communications, $V$ Conférence International ICAZ, Bordeaux, 1 p.

Colomer, F., Gaete, R., Galobart, A., Maroto, J., Menéndez, E. i Ros, X. 1992. El jaciment paleontològic d'Incarcal (Crespià. Pla de l'Estany). El medi natural del Vallès, 3, III Col•loqui de Naturalistes Vallesans, Sabadell, 26-32.

Corominas, J. M. y Marqués, J. 1967. La comarca de Bañolas, fasc. I, Catálogo Monumental de la Provincia de Gerona, Gerona, 153 pp.

Cortada, T. i Maroto, J.1990. La dent humana paleolítica de la cova Mollet I (Serinyà). Quaderns del Centre d'Estudis Comarcals de Banyoles, 1988-1989, 135-148.

Crane, H. R. and Griffin, J. B. 1960. University of Michigan Dates V. Radiocarbon, 2, 148.

Deckker, P. de, Geurts, M. A. and Julià, R. 1979. Seasonal rhythmites from a Lower Pleistocene lake in Northeastern Spain. Paleography, Paleoclimatology, Paleoecology, 26, 43-71.

Delibrias, G., Roman, O. i Le Hasif, G. (1987). Datation parle méthode du carbone 14 du reinplisage de la grotte de l'Arbreda. Gypsela, VI, 133-135.

Estévez, J. 1977-78. Un percutor solutrense en asta de reno hallado en Serinyà (Girona). Pyrenae, 13-14, pp. 301-305.

Estévez, J. 1978. Primer hallazgo del buey almizclado (Ovibos moschatus, Zimmerman) en el Pleistoceno peninsular. Acta Geológica Hispánica, XIII, 2, pp. 59-60.

Estévez, J. 1980. El aprovechamiento de los recursos faunísticos: aproximación a la economía en el Paleolítico catalán. Cypsela, III, 9-30.

Estévez, J. 1985. La fauna paleolítica de la cova de l'Arbreda. Quaderns del Centre d'Estudis Comarcals de Banyoles, 1980-1985, 27-30.

Estévez, J. 1987. La fauna de l'Arbreda (sector Alfa) en el conjunt de les faunes del Paleolític superior. Cypsela, VI, 73-87.

Galobart, A., Maroto, J., Menéndez, E., Ros, X., Gaete, R. y Colomer, F. 1990. El yacimiento del Pleistoceno Inferior de Incarcal (Crespià, Girona). Com. Reunión de Tafonomía y Fosilización, 161-167.

Julià, R. 1977. Nuevos datos sobre la posición cronoestratigráfica de los materiales cuaternarios de la cuenca lacustre de Banyoles-Besalú (Girona). Acta Geológica Hispánica, XII, 1/3, 55-59.

Julià, R. 1979. El cuaternario de la cuenca lacustre Banyoles-Besalú. Actas de la IV Reunión del Grupo de Trabajo del Cuaternario, Julià, R., Marqués, M. A., Mir, A., Serrat, D. y Gallart, F. (eds.), Banyoles, 279-287.

Julià R. 1980. La conca lacustre de Banyoles-Besalú, Centre d'Estudis Comarcals de Banyoles, 187 pp.

Julià, R. et Suc, J. P. 1980. Analyse pollinique des dépôts lacustres du Pléistocène inférieur de Banyoles (Bañolas, site de la Bòbila Ordis -Espagne): un élément nouveau dans la reconstitution de l'histoire paléoclimatique des régions méditerranéennes d'Europe occidentale. Géobios, 13, 1, 5-19.
Julià, R. y Villalta, J. F. de 1974. El Ampurdán. Coloquio internacional sobre bioestratigrafía continental de Neógeno superior y Cuaternario inferior, guía 28.9, Aguirre, E. (ed.), Montpellier-Madrid, 3-9.

Julià, R. y Villalta, J. F. de 1984. El yacimiento de vertebrados del Pleistoceno inferior de Crespià (Girona, NE de la Península Ibérica). Acta Geológica Hispánica, 19, 2, 129-138.

Julià, R. and Bischoff, J. L. (1991). Radiometric dating of quaternary deposits and the hominid mandibule of Lake Banyolas. Spain. Journal of Archeological Science, 18, London, 707-722.

Julià, R., Maroto, J. y Soler, N. 1987. La mandíbula de Banyoles: antecedents i context de la seva troballa. Cypsela, VI, 43-52.

Maroto, J. (ed.) 1993. La mandíbula de Banyoles en el context dels fòssils humans del pleistocè, Sèrie monogràfica, 13, Centre d'Investigacions Arqueològiques, Girona, 198 pp.

Maroto, J. 1994. El pas del paleolític mitjà al paleolític superior a Catalunya i la seva interpretació dins del context geogràfic franco-ibèric, Tesi doctoral, Universitat de Girona.

Maroto, J. i Galobart, A. 1992. Incarcal (Crespià, Pla de l'Estany): un jaciment representatiu de la fauna de grans mamífers del plistocè inferior. Tribuna d'Arqueologia, 1990-1991, 7-15.

Maroto, J. i Soler, N. 1985. Un elefant d'un milió d'anys trobat a Crespià, Revista de Girona, 110, 52-54.

Maroto, J., Soler, N., y Mir, A. 1987. La cueva de Mollet I (Serinyà, Gerona). Cypsela, VI, 73-87.

Mir, A. 1979. La fauna de la Cova d'En Mollet I, Serinyà (Girona) procedente de las campañas de excavación 1947-1972. Actas de la IV Reunión del Grupo de Trabajo del Cuaternario, Julià, R., Marqués, M. A., Mir, A., Serrat, D. y Gallart, F. (eds.), Banyoles, pp. 166-170.

Mir, A. y Salas, R. 1976. Tres nuevos carnívoros del yacimiento cuaternario de la Cova d'en Mollet I, Serinyà (prov. de Girona). Instituto de Investigaciones Geológicas, XXXI, Universidad de Barcelona, 97-124.

Nadal, J., Albert, R. M. y Juan, J. (en prensa). Nuevas aportaciones arqueozoológicas y arqueobotánicas del yacimiento magdaleniense de la Bora Gran d'en Carreras (Serinyà, Pla de l'Estany). El món mediterrani després del pleniglacial.

Roiron, P. 1983. Nouvelle étude de la macroflore plio-pléistocène de Crespià (Catalogne-Espagne), Geobios, 16, 6, 687-715.

Sanz, E., Comas, P. i Maroto, J. 1987. Inventari paleontològic del fons de Museu Arqueològic Comarcal de Banyoles procedents de la zona Banyoles-Mata i adjacents. Quaderns del Centre d'Estudis Comarcals de Banyoles, 1986-1987, 103-107.

Soler, N. i Maroto, J. (1987). Els nivells d'ocupació del Paleolític Superior a la cova de l'Arbreda (Serinyà, Girona). Gipsela. VI, 221-228.

Torres, T. de 1988. Osos (Mammalia, Carnivora, Ursidae) del Pleistoceno de la Península Ibérica, Publicaciones Especiales del Boletín Geológico y Minero, Instituto Geológico y Minero de España, Madrid, 316 pp.

Villalta, J. F. de y Llompart, C. 1981. Hallazgo de un molar de Mammut borsoni (Hays) en el Plioceno del Baix Empordà (Prov. Girona). Acta Geológica Hispánica, 16, 4, 195-197.

Villalta, J. F. y Vicente, J. 1972. Una flora del Cuaternario antiguo en las cercanías de Crespià. Acta Geológica Hispánica, VII, 4, 120-128.

Manuscrito recibido: 12 de diciembre, 1994 Manuscrito aceptado: 6 de noviembre, 1995 